\title{
A new Channel- and QoS-Aware Scheduling Scheme for Real-time Services in LTE Network
}

\author{
Duy-Huy Nguyen \\ SAMOVAR, Télécom SudParis, CNRS, \\ Université Paris-Saclay \\ 9 rue Charles Fourier - 91011 Evry Cedex
}

\author{
Hang Nguyen \\ SAMOVAR, Télécom SudParis, CNRS, \\ Université Paris-Saclay \\ 9 rue Charles Fourier - 91011 Evry Cedex
}

\author{
Éric Renault \\ SAMOVAR, Télécom SudParis, CNRS, \\ Université Paris-Saclay \\ 9 rue Charles Fourier - 91011 Evry Cedex
}

\begin{abstract}
This paper proposes a new scheduling scheme which is based on Channel- and QoS-Aware for real-time traffics in LTE downlink direction. The real-time services (VoIP, Video, etc.) are very sensitive to network impairments such as delay, packet loss, jitter, etc. The proposed scheduling scheme considers Maximum Queue Size (MQS) as an essential factor for the priority metric and the combination of well-known M-LWDF-based algorithms such as M-LWDF, VT-MLWDF and Queue-HOL-MLWDF. The simulation results show that the proposed scheme has the performance which not only satisfies QoS requirements of realtime services but also outperforms the M-LWDF, VT-MLWDF and Queue-HOL-MLWDF schedulers in terms of Packet Loss Rate (PLR), cell throughput, Fairness Index (FI) and Spectral Efficiency (SE), especially for Video flow. The proposed scheduler also significantly improves the delay in comparison with the VT-MLWDF and Queue-HOL-MLWDF schedulers ones for both VoIP and Video flows. The performance evaluation is compared in terms of Delay, PLR, Throughput, FI and SE for MLWDF, VT-MLWDF, Queue-HOL-MLWDF and the proposed schedulers.
\end{abstract}

\section{Keywords}

Scheduling Algorithm, Resource Allocation, Real-time Services, LTE, QoS

\section{INTRODUCTION}

The Long Term Evolution (LTE) is developed by the Third Generation Partnership Project (3GPP) [1]. It is a mobile network which has high data rate, low delay and fully packet-based. This means to improve the capability of legacy system by increasing data rates and extending superior QoS for various multimedia applications. Basic components of LTE network include a powerful eNodeB (eNB) station and several User equipments (UEs) in addition to a gateway [5]. The eNB station combines with core network through several standard complicated protocols. Basic packet scheduling is carried out by the network operator in both UE and eNB station for both uplink as well as downlink. However, according to the $3 \mathrm{GPP}$, there are no firm specifications for scheduling technique in LTE network. One of the most important modules of packet scheduling is Radio Resource Management (RRM) which decides users that would transmit their data on the air interface. The packet scheduling should integrate fairness in terms of throughput as well as the service policies to which users subscribe [4].

LTE architecture defines a complete table with packet delay budget and packet loss rates for performing different service class in the network [1] which some or all may be executed by service provider. This table divides resource types in the LTE network into two groups, those are Guaranteed Bit Rate (GBR) and Non-GBR. In order to meet different QoS requirements for these groups, several packet scheduling algorithms have been proposed. According to [7], the scheduling strategies for LTE downlink are divided into five groups including: (1) Channel-unware strategies, (2) Channel-aware/QoS-unaware strategies, (3) Channel-aware/QoS-aware strategies, (4) Semipersistent scheduling for VoIP support, and (5) Energy-aware strategies. For the real-time services such as VoIP, Video, etc. which are very sensitive to delay and PLR. So that, the Channelaware/QoS-aware strategies are very essential for them. Several well-known scheduling algorithms for this group as the Modified Largest Weighted Delay First (M-LWDF) [6], the Exponential/PF (EXP/PF) [16], the LOG and EXP rules [17] or the Frame Level Scheduler (FLS) [14]. In these schedulers, there is only the FLS which guarantees bounded delay for real-time flows, the remaining schedulers transmits users data in a given Transmission Time Interval (TTI) by assigning a computed priority metric that is specific to the scheduler functionality. However, due to the lack of delay budget and PLR attribute, these schedulers are not suitable for supporting simultaneously real-time and non realtime traffic [5].

In this paper, we assess the performance of the proposed scheduler and the M-LWDF, Virtual token M-LWDF (VT-MLWDF) and Queue Head of Line packet delay M-LWDF (Queue-HOLMLWDF) schedulers for real-time services such as VoIP and Video. The proposal is formed from the idea in [18]. The authors in [18] proposed a new scheduling scheme for real-time communication in LTE networks by modifying several M-LWDF-based schedulers. We extend the idea in [18] and propose to consider the MQS as an essential and effective factor for the priority metric. This factor has significant effects on the system performance. In the LTE-Sim [13], this factor is fixed equal to 0. This means the MQS is infinite. So that, in the scheduling process, the MQS is not considered. However, in fact, the MQS should be a finite value because if the MQS value is infinite then the delay will increase and the congestion could be increased. Therefore, the MQS needs to be considered as an essential factor in the metric of the scheduling algorithms. Besides, the combination and essential modifications of the M-LWDF, VT-MLWDF and QueueHOL-MLWDF schedulers is also considered in the metric in the 
proposed scheduler. This paper presents a new Channel- and QoS-aware scheduling scheme for downlink direction in LTE systems. The simulation results were implemented in the open source LTE system simulator called Long Term Evolution-Sim (LTE-Sim) [13] and are compared in terms of Delay, PLR, Cell throughput, FI and SE for the number of user from 5 to 25

The remainder of this paper is organized as follows: Overview of scheduling in LTE downlink is described in section 2 In section 3. we present the proposed scheduling scheme. The simulation results and performance evaluation of the proposed scheduler are analysed in section 4 The conclusion and future work is represented in section 5

\section{SCHEDULING IN LTE DOWNLLINK}

\subsection{System traffic}

Figure 1 represents the structure of LTE frame for the downlink air interface [11]. A frame is $10 \mathrm{~ms}$ in length and is divided into 10 sub-frames in time domain. So that, each sub-frame is $1 \mathrm{~ms}$ in length and is divided into 2 slots where each slot is $0.5 \mathrm{~ms}$ in length. In frequency domain, each slot is divided into a number of resource blocks (RBs) (from 6 to $10 \mathrm{RBs).} \mathrm{Each} \mathrm{slot} \mathrm{contains}$ 6 or 7 Orthogonal Frequency Division Multiplexing (OFDM) symbols in normal cyclic prefixes and extended cyclic prefixes, respectively. Each time slot in frequency domain is divided into bands of $180 \mathrm{kHz}$ which contains 12 consecutive sub-carriers. Each RB is a basic unit of exchanging information in the downlink direction of LTE system. So that, a RB is the radio resource that is available for a user in LTE network and is defined by both frequency and time domains. The number of RBs in a slot depends on the bandwidth of the LTE system [19]. Each sub-frame represents a TTI which is the minimum transmission unit. Each TTI contains at least one transport block per UE. The RB size is the same one for all bandwidths [3].

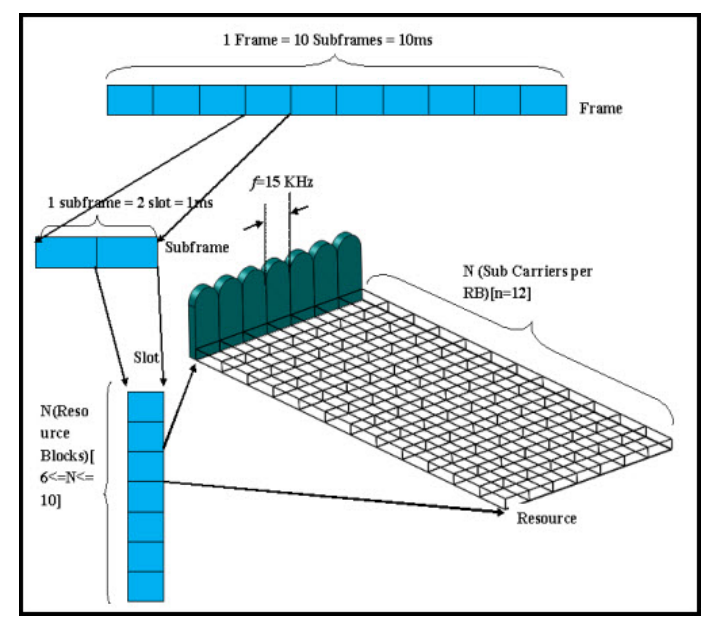

Fig. 1. Resource grid

Figure 2 represents the main RRM modules that interact with the downlink packet scheduler. The entire process can be divided into a sequence of tasks which are repeated in each TTI [7]. When packets arrive at the buffer in the eNB, they are given a time stamp and are queued for transmission. For each packet in the queue, the Head of Line packet delay (HOL) and the queue length (QL) are estimated. If the HOL packet delay or QL exceeds specified thresholds for the flow, then the packet will be discarded. The packet scheduler decides which users will be served according to a scheduling algorithm based on the metric of the corresponding algorithm. In order to have a suitable scheduling scheme, we have to have the trade-off between channel state, QoS requirements and queue status. In the proposed scheduling scheme, when the scheduler making the decisions, the instantaneous or average channel conditions, HOL packet delay, status of receiving buffer such as QL and MQS or type of service are being used. The scheduling process in Figure 2 is described as followings:

-Each UE decodes the reference signals, calculates the Channel Quality Indicator (CQI) and sends it back to the eNB.

- The eNB utilizes the CQI information for the allocation decisions and fills up a RB "allocation mask".

-The Adaptive Modulation and Coding (AMC) module selects the best Modulation and Coding Scheme (MCS) that should be used for the data transmission by scheduled users.

- The information about these users, the allocated RBs, and the selected MCS are sent to the UEs on the Physical Downlink Control Channel (PDCCH).

-Each UE reads the PDCCH payload and in case it has been scheduled, accesses to the proper Physical Downlink Shared Channel (PDSCH) payload.

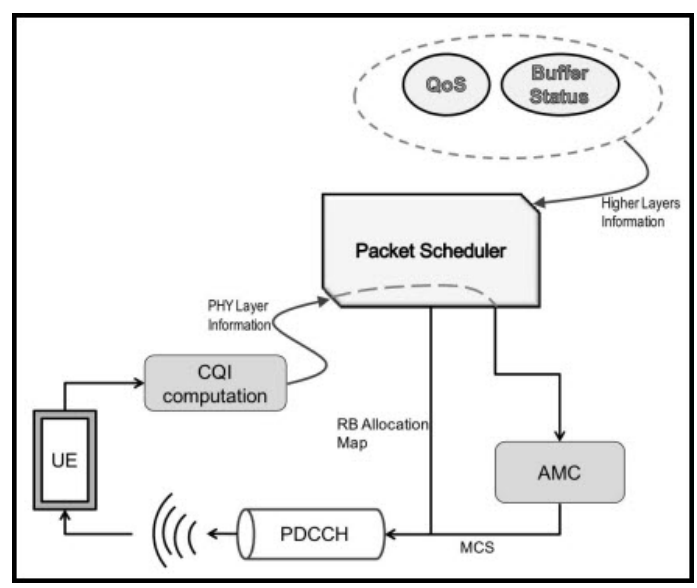

Fig. 2. Simplied model of a packet scheduler

\subsection{Scheduling algorithms}

The purpose of scheduling algorithm in LTE is to maximum system performance [8]. The chosen UE is suitable for scheduling decisions. In order to make scheduling decisions, there is much information included, such as the number of sessions, their served rates, link states, and the statuses of session queues [8]. The eNB offers a scheduling decision based on the the Channel quality Indicator (CQI) which is sent to the eNB from the UE. The CQI is then exploited by the scheduler link adaptation module to select an UE with the most suitable modulation scheme and coding rate at the PHY layer with the objective of the spectral efficiency maximization.

We assume that the metric assigned to the stream $i$ on $j$-th subchannel is noted by $w_{i, j}$. In order obtain the metric, the scheduler usually need to know the average transmission rate $\left(\bar{R}_{i}\right)$ of flow $i$, and the flow rate available to the UE on the $j$-th sub-channel. In particular, at each TTI, the estimate $\bar{R}_{i}$ is given by [13]:

$$
\bar{R}_{i}(k)=0.8 \times \bar{R}_{i}(k-1)+0.2 \times \bar{r}_{i}(k)
$$

Where:

$-\bar{R}_{i}(k-1)$ : The average transmission data rate estimating at the $(k-1)$-th TTI.

$-\bar{r}_{i}(k)$ : The rate allocated to $i$-th flow during the $k$-th TTI 
In the following subsections, we will describe the metric of several well-known M-LWDF based scheduling algorithms which are related to the proposed scheduling scheme including: MLWDF, VT-MLWDF and Queue-HOL-MLWDF schedulers.

2.2.1 The M-LWDF scheduler. The M-LWDF scheduling algorithm is used to support multiple real-time services in CDMAHDR systems [6]. For each real-time flow, by considering the maximum time $\tau_{i}$, the probability is defined as the maximum probability $\delta_{i}$ which is the time of the first packet of the queue exceeds the fixed maximum time $D_{H O L, i}$. In this algorithm, the metrics for real-time and non real-time services are different. In order to offer priority to real-time flows, the metric is given as follows:

$$
w_{i, j}=\alpha_{i} \times D_{H O L, i} \times \frac{r_{i, j}}{R_{i}}
$$

Where:

$-r_{i, j}$ : The rate assigned to $i$-th flow during the $k$-th TTI

$-\bar{R}_{i}$ : The average transmission data rate estimating

$-\alpha_{i}$ : A factor and is given by: $\alpha_{i}=-\frac{\log \left(\delta_{i}\right)}{\tau_{i}}$

2.2.2 The VT-MLWDF scheduler. The main purpose of this scheduling algorithm is to improve the QoS performance metrics for real-time communications such as Video, VoIP, Gaming, etc. and to maintain minimum throughput for non real-time services [10]. The idea of this scheduling algorithm is replacement of $D_{H O L, i}$ factor in the M-LWDF scheduling algorithm on $Q_{i}$ factor. The metric of VT-MLWDF scheduler if represented as following equation:

$$
w_{i, j}=\alpha_{i} \times Q_{i} \times \frac{r_{i, j}}{R_{i}}
$$

Where:

$-r_{i, j}$ and $\bar{R}_{i}$ are similar to the description in previous equation. $-Q_{i}$ : The token queue length (a counter value at time $t$ )

2.2.3 The Queue-HOL-MLWDF scheduler. The main goal of this scheduling algorithm is to enhance the QoS performance parameters [12]. This algorithm adds the queue size into the metric of the M-LWDF algorithm. This is the combination between two M-LWDF and VT-MLWDF algorithms. In order to measure the performance of this algorithm when serving the real-time flows, the metric used in this algorithm is performed as follows:

$$
w_{i, j}=\alpha_{i} \times D_{H O L, i} \times Q_{i} \times \frac{r_{i, j}}{\bar{R}_{i}}
$$

Where: $\alpha_{i}, D_{H O L, i}, Q_{i}, r_{i, j}$ and $\bar{R}_{i}$ are similar to the descriptions in the above equations.

\section{THE PROPOSED SCHEDULING SCHEME}

In the proposed scheduling scheme, we consider the characteristics of the real-time services such as VoIP, Video, etc. These services are sensitive to packet loss and delay, thus, scheduling process should consider various factors. In the related scheduling algorithms above, the authors almost focused on Head of Line (HOL) packet delay, virtual token length besides other factors such as $\alpha_{i}, r_{i, j}$ and $\bar{R}_{i}$. According to our knowledge, there are no articles which mention about the MQS. We think that, this factor has strong effects on the system performance. In the LTE-Sim [13], this factor is fixed equal to 0 . This means the MQS is infinite. Hence the MQS is not considered in the scheduling process. However, in fact, the MQS should be finite. If the MQS value is infinite then the delay will increase and the congestion could be thus increased. Therefore, the MQS should be considered as a necessary factor in the metric of the scheduling algorithms.

The main idea of the proposed scheduling algorithm is the consideration of the MQS factor (called also $Q_{i, \max }$ ) included into the metric of the scheduling algorithm by remaining queue size
$\left(Q_{i, \max }-Q_{i}\right)$. This means the lower $\left(Q_{i, \max }-Q_{i}\right)$ value, the higher priority for the UE. Besides, in order to improve the system performance, we also consider the deadline is a factor of the metric and essential some modifications. According to [5], the remaining time $\left(\tau_{i}-D_{H O L, i}\right)$ which is assigned a deadline for each flow that has a packet queued at the eNB. It can be said that, the proposed scheduling scheme is the combination of the MQS factor and the modifications of the metrics of the M-LWDF, VTMLWDF and Queue-HOL-MLWDF schedulers which are described above. The metric in the proposed scheduling scheme is defined as follows:

$$
w_{i, j}=\delta_{i} \times\left(Q_{i, \max }-Q_{i}\right) \times \frac{D_{H O L, i}}{\tau_{i}-D_{H O L, i}} \times \frac{r_{i, j}}{\bar{R}_{i}}
$$

Where:

$-\delta_{i}, Q_{i}, D_{H O L, i}, \tau_{i}, r_{i, j}$ and $\bar{R}_{i}$ have the same significances in the previous formulas.

$-Q_{i, \max }$ : The MQS of the user $i$. This value can be obtained in bytes through some functions in LTE-Sim [13].

The reason of choosing the remaining time $\left(\tau_{i}-D_{H O L, i}\right)$ is that in Equation 5, the $\alpha_{i}$ is not used due to it is calculated according to the $\tau_{i}$ such as described previously. In addition, according to [5], the lower $\left(\tau_{i}-D_{H O L, i}\right)$ value, the higher priority for UE. So that, the proposed metric for scheduling algorithm has more QoS ties result in the proposed scheduling scheme outperforms several other related scheduling algorithms when compared to. The steps of the proposed scheduling scheme are described as follows:

-Step 1: Identify real-time or any other traffic is queuing in the buffer

-Step 2: Determine the user metrics $\left(Q_{i, \max }, Q_{i}\right)$ and other parameters. Calculate the scheduling metric $\left(w_{i, j}\right)$ according to the Equation 5

-Step 3: Find the user having the metric that is met the scheduling criteria

-Step 4: Count the set of available RBs to allocate which RBs to the chosen users

-Step 5: Assign the resource block $\mathrm{R}^{*}$ to the user $\mathrm{U}^{*}$ which has the satisfied metric

-Step 6: Schedule the user $U^{*}$ first

-Step 7: Delete the user $\mathrm{U}^{*}$ and resource block $\mathrm{R}^{*}$ from their corresponding lists

-Step 8: Repeat the steps from 1 to 7 until all users are scheduled.

\section{SIMULATION ENVIRONMENT AND PERFORMANCE EVALUATION}

\subsection{Simulation environment}

4.1.1 Simulation parameters. In this paper, we investigate the performance of the M-LWDF, VT-MLDF, Queue-HOLMLWDF and the proposed schedulers in LTE downlink direction. The simulation process is performed in a single cell with interference with the number of users in the interval [5, 25]. Users move randomly at a speed of $3 \mathrm{~km} / \mathrm{h}$ and each user uses a VoIP flow as well as a Video one. The users using best-effort flows have not been considered.

In order to evaluate the system performance, we used LTE-Sim [13]. This is a open source framework for researchers and academic community. The basic parameters used in the simulation are represented in Table 4.1.1 
Table 1. Simulation parameters

\begin{tabular}{|l|l|}
\hline Simulation Parameters & \multicolumn{1}{|c|}{ Values } \\
\hline Simulation duration & $100 \mathrm{~s}$ \\
\hline Frame structure & FDD \\
\hline Cell radius & $1 \mathrm{~km}$ \\
\hline Bandwidth & $5 \mathrm{MHz}$ \\
\hline Video bit-rate & $242 \mathrm{kbps}$ \\
\hline VoIP bit-rate & $8 \mathrm{kbps}$ \\
\hline User speed & $3 \mathrm{~km} / \mathrm{h}$ \\
\hline Number of users & $5,10,15,20,25$ UEs \\
\hline Maximum delay & $0.1 \mathrm{~s}$ \\
\hline MQS & $10^{5}$ bytes \\
\hline
\end{tabular}

4.1.2 Traffic model. In the simulation scenario, the eNB is located at the center of the macrocell using an ommi-directional antenna in a $5 \mathrm{MHz}$ bandwidth. Each UE uses a VoIP flow and a Video flow at the same time. For the VoIP flow, a G.729 voice stream with a bit-rate of $8 \mathrm{kbps}$ was considered. The voice flow is a bursty application that is modeled with an ON/OFF Markov chain [9].

For the video flow, a trace-based application that generates packets based on realistic video trace files with a bit-rate of $242 \mathrm{kbps}$ was used in [15] and it is also available in [13]. In order to obtain a realistic simulation of an H.264 SVC video streaming, we used an encoded video sequence "foreman.yuv", which is publicly available.

The LTE propagation loss model is formed by four different models including: Path loss, Multipath, Penetration and Shadowing [2].

-Path loss: $P L=128.1+37.6 \times \log (d)$, with $\mathrm{d}$ is the distance between the UE and the eNB in $\mathrm{km}$.

-Multipath: Jakes model

-Penetration loss: $10 \mathrm{~dB}$

-Shadowing: Log-normal distribution with mean $0 \mathrm{~dB}$ and standard deviation of $8 \mathrm{~dB}$.

\subsection{Performance evaluation}

In order to evaluate the performance of the scheduling scheme, we use the LTE-Sim [13]. The simulation results are compared to the ones of the other schedulers including the M-LWDF, VTMLWDF and Queue-HOL-MLWDF. The performance evaluation is performed in terms of delay, PLR, cell throughput, FI and SE. The analysis of the simulation results are represented in the following subsection.

4.2.1 Delay. Figure 3 shows the delay of VoIP flow. It is clear that, the proposed scheduler has the lower delay than the QueueHOL-MLWDF and the VT-MLWDF schedulers. For the number of VoIP user from 5 to 15 , the proposed scheduler has the delay as same as the M-LWDF one. Otherwise, the M-LWDF scheduler has the lower delay then the proposed scheduler when the number of VoIP user more than 15 UEs.

For the Video flow, such as shown in the Figure 4 the proposed scheduler has the lower delay than the VT-MLWDF and QueueHOL-MLWDF schedulers when the number of Video user from 5 to 23 UEs. The proposed scheduler has the same delay as the M-LWDF scheduler when the number of Video user from 5 to 15 UEs. However, when the number of Video user more than 15 UEs, the M-LWDF scheduler has the lower delay than the proposed scheduler one.

It can be said that for the number of UE from 5 to 15 , the proposed scheduler has the same delay when compared to the MLWDF scheduler and has the lower delay in comparison with the remaining schedulers.

4.2.2 Packet Loss Rate. Figure 5 represents the PLR of VoIP flow. Although the proposed scheduler does not have the good

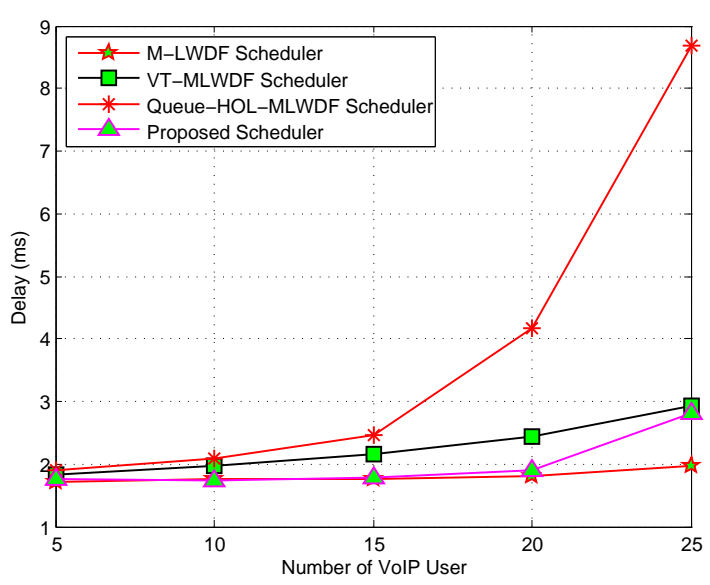

Fig. 3. Delay vs number of VoIP user

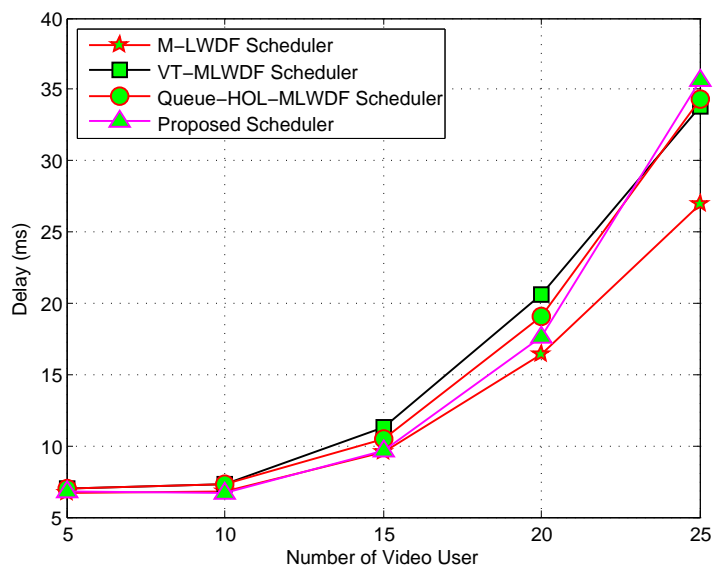

Fig. 4. Delay vs number of Video user

PLR when compared to the three remaining schedulers, however the proposed scheduler has the lower PLR for the number of VoIP user from 8 to 14 in comparison with the M-LWDF scheduler. Especially, when the number of VoIP user more than or equal to 20 , the proposed scheduler has the stable PLR. This is very good when the number of VoIP user more than 25 UEs.

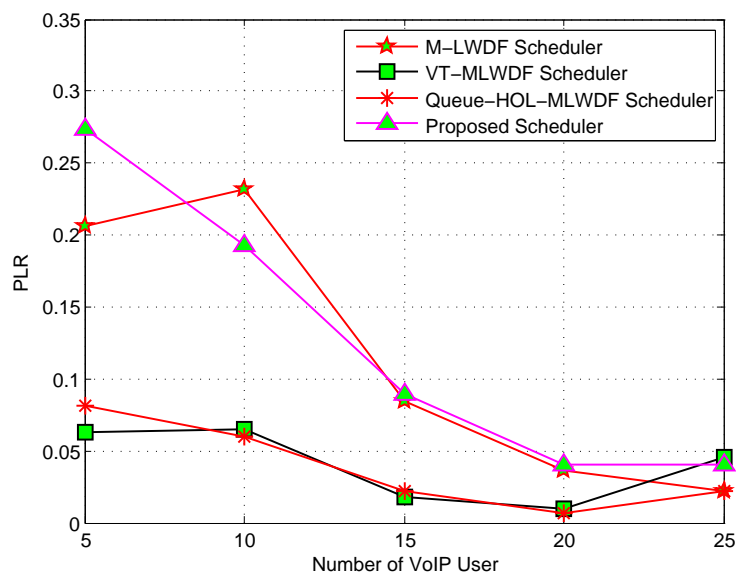

Fig. 5. Packet loss rate vs number of VoIP user 
For the Video flow, such as shown in the Figure 6, although the proposed scheduler does not has good PLR when the number of Video user less than 10, when the number of Video user from 10 to 15 , the proposed scheduler has the PLR as same as the MLWDF scheduler one. Specially, the proposed scheduler has the lowest PLR when the number of Video user more than 15 UEs when compared to the remaining schedulers. It can be said that the proposed scheduler is very efficient for the PLR of Video flow when the number of Video user more than 15 UEs.

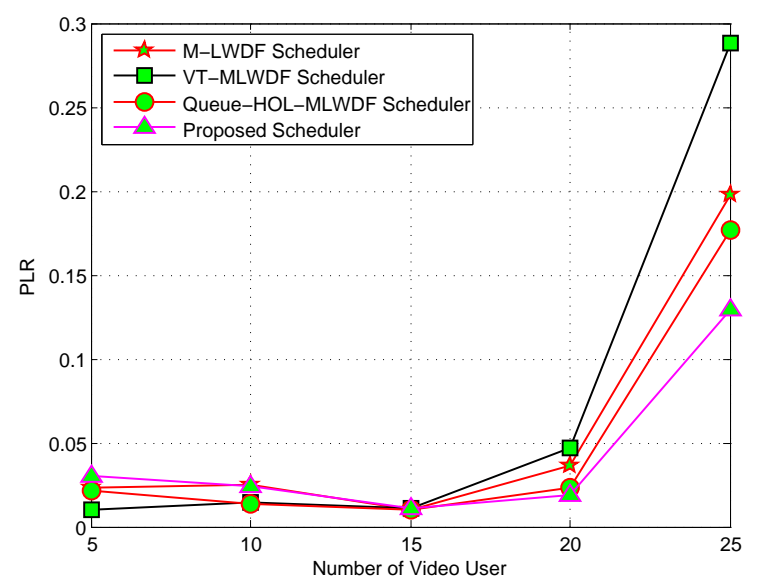

Fig. 6. Packet loss rate vs number of Video user

4.2.3 Cell throughput. For the VoIP flow, as shown in the Figure 7 the proposed scheduler has the cell throughput as approximate as the remaining schedulers, except the Queue-HOLMLWDF scheduler has the highest cell throughput when the number of VoIP user from 5 to 8 UEs, the proposed scheduler has the highest cell throughput when the number of VoIP user around 15 UEs. It is clear that the M-LWDF has the lowest cell throughput when the number of VoIP more than 20 UEs.

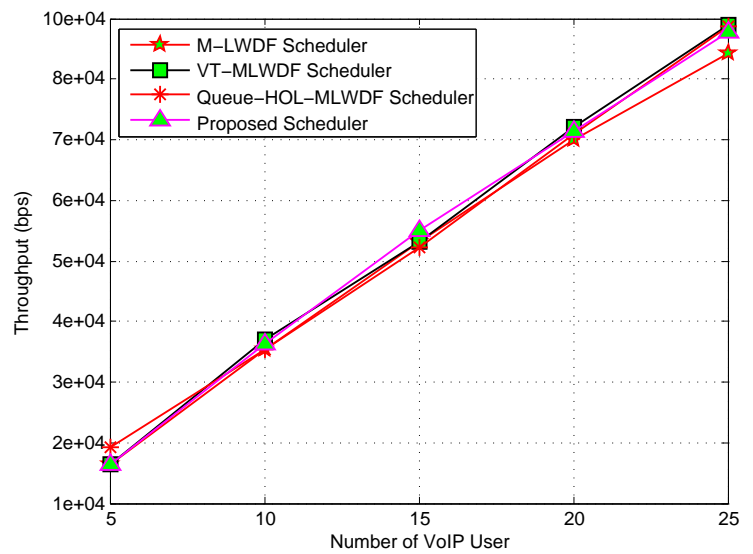

Fig. 7. Throughput vs number of VoIP user

Figure 8 shows the cell throughput of Video flow. The proposed scheduler has the cell throughput as same as the three remaining schedulers when the number of Video user from 5 to 20 UEs. When the number of Video user more than 20, the proposed scheduler has the highest cell throughput in comparison with the M-LWDF, VT-MLWDF and Queue-HOL-MLWDF schedulers.

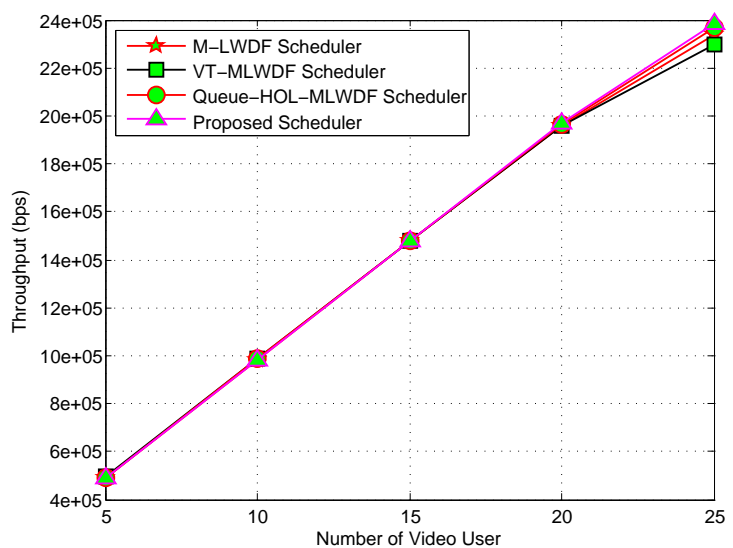

Fig. 8. Throughput vs number of Video user

4.2.4 Fairness index. Figure 9 shows the FI of VoIP flow. The proposed scheduler has the FI as same as the M-LWDF and VTMLWDF schedulers one and higher than Queue-HOL-MLWDF when the number of VoIP from 5 to 15 UEs. Although when the number of VoIP is greater than 17, the proposed scheduler has the lowest FI when compared to the remaining schedulers, but it seems that the proposed scheduler will have the FI strongly increased when the number of VoIP user more than 25 UEs.

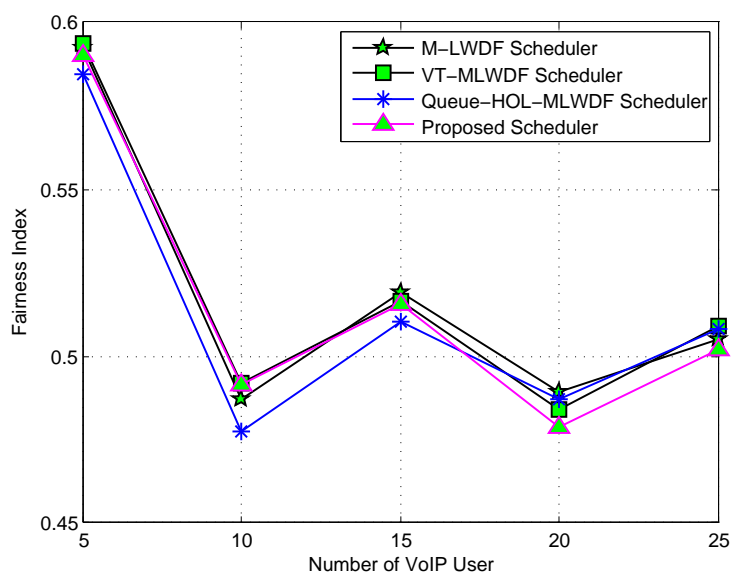

Fig. 9. Fairness Index vs number of VoIP user

For the Video flow, as shown in the Figure 10 the proposed scheduler has the same FI when compared to the remaining schedulers for the number of Video user from 5 to 20 . When the number of Video user is more than 20, the proposed scheduler has the highest FI in comparison with the M-LWDF, VTMLWDF and Queue-HOL-MLWDF schedulers ones.

4.2.5 Spectral efficiency. Such as shown in the Figure 11 the proposed scheduler has the SE as same as the remaining schedulers when the number of user from 5 to 20 . When the number of user is more than 20, the proposed scheduler has the highest SE when compared to the M-LWDF, VT-MLWDF and QueueHOL-MLWDF schedulers ones.

4.2.6 Effects of MQS on the system performance. In order to evaluate the effects of the MQS on the system performance, we simulate the proposed scheduler with the different MQS values and compare in terms of Delay, PLR and Cell throughput. Figure 12 shows the effect of the MQS on the delay of VoIP flow. The 


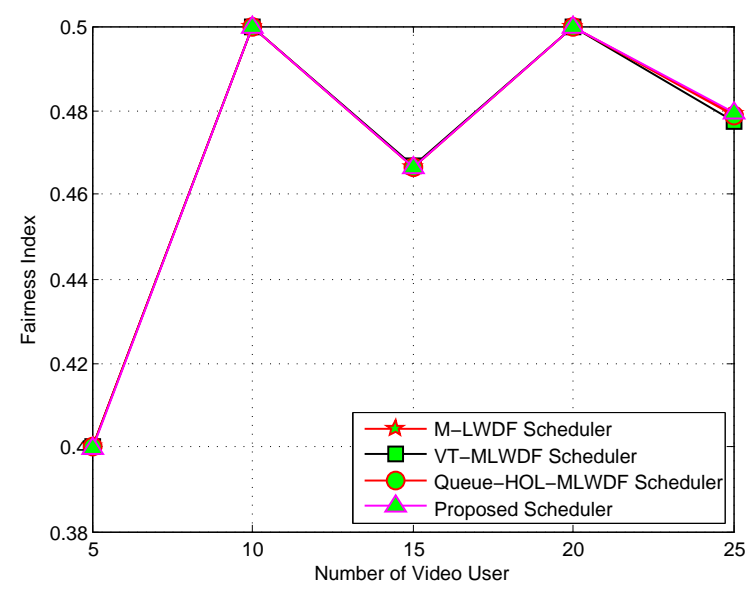

Fig. 10. Fairness Index vs number of Video user

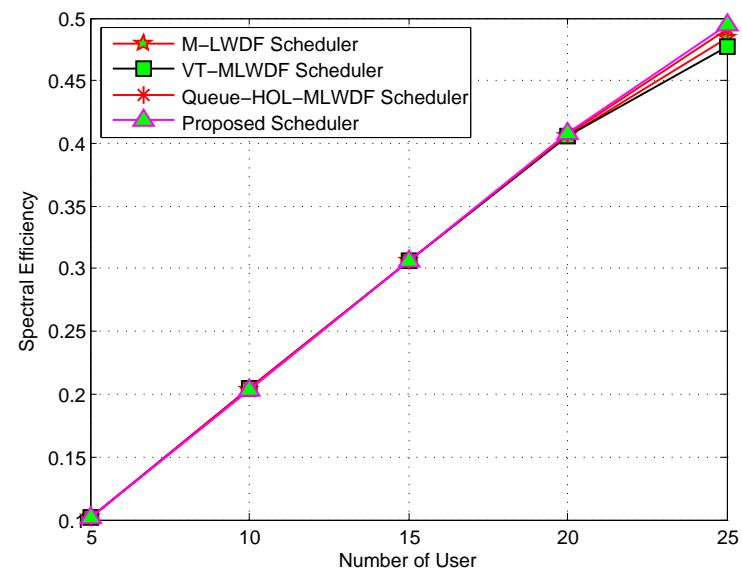

Fig. 11. Spectral efficiency vs number of VoIP user

simulation result shows that the lower MQS, the lower delay for VoIP flow.

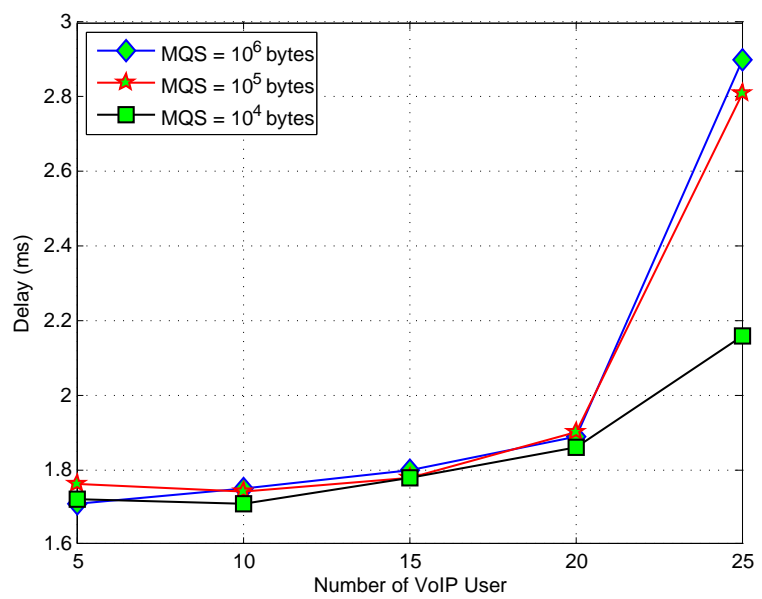

Fig. 12. The effect of the MQS on the delay of VoIP flow

For the Video flow as shown in Figure 13 , the proposed scheduler has the lowest delay for the MQS equal to $10^{4}$ bytes. For the
MQS equal to $10^{5}$ or $10^{6}$ bytes, the delay of Video flow is nearly the same.

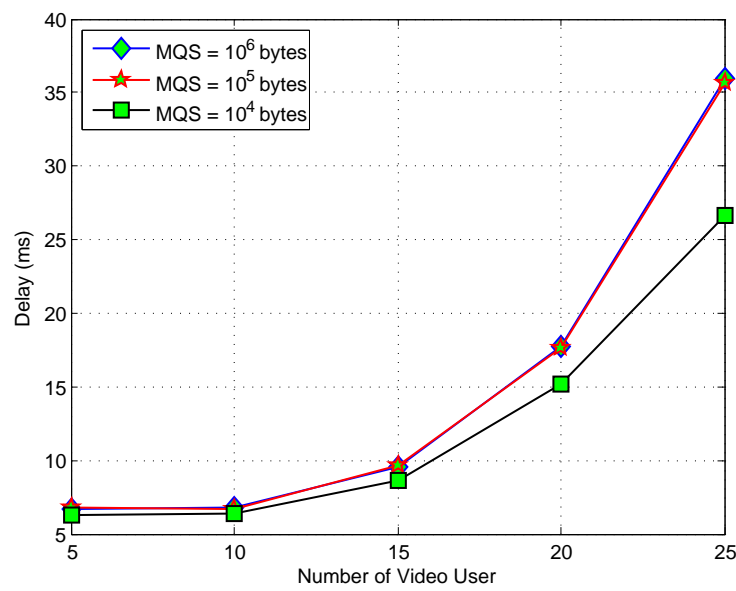

Fig. 13. The effect of the MQS on the delay of Video flow

Figure 14 represents the effect of the MQS on PLR of VoIP flow. As the result, there is not any MQS which offers the lowest PLR. However, when the number of VoIP users is more than 20, the proposed scheduler with the MQS $=10^{5}$ bytes has the stable PLR. This demonstrates that in this case, the PLR may be also stable when the number of VoIP users more than 25 .

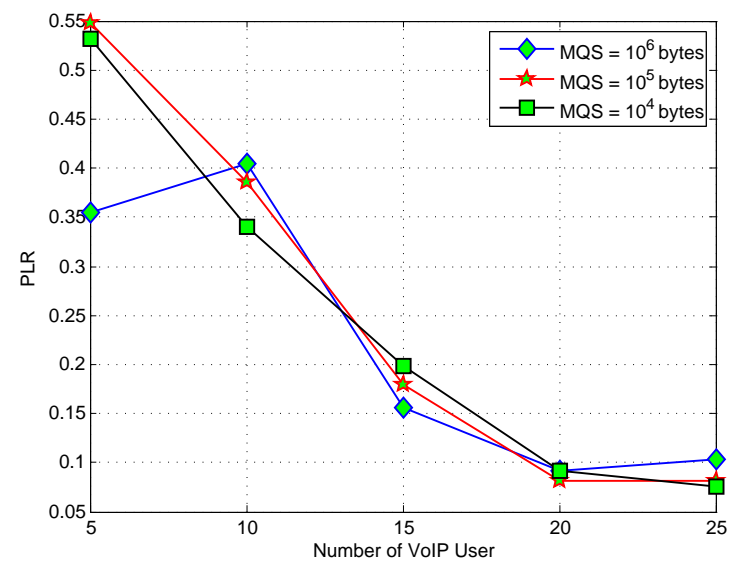

Fig. 14. The effect of the MQS on the PLR of VoIP flow

For the Video flow, the effect of the MQS on the PLR is represented in Figure 15 . The result shows that the proposed scheduler has the same PLR with the MQS equals $10^{5}$ or $10^{6}$ bytes when the number of Video user is more than or equal to 10 . For the MQS is equal to $10^{4}$ bytes, the PLR has the highest PLR which is about 10 times than the MQS is equal to $10^{5}$ or $10^{6}$ bytes.

Figure 16 shows the effect of the MQS on cell throughput of VoIP flow. In general, the proposed scheduler has the highest cell throughput when the MQS equal to $10^{4}$ bytes and has the approximate one in comparison with the MQS equal to $10^{5}$ bytes. In case of the MQS equals $10^{6}$ bytes, the proposed scheduler has the lowest cell throughput.

For the Video flow, the effect of the MQS on the cell throughput is represented in Figure 17 The result show that the proposed scheduler has the same cell throughput for the MQS equals $10^{5}$ or $10^{6}$ bytes and has the lowest cell throughput for the MQS equals $10^{4}$ bytes. 


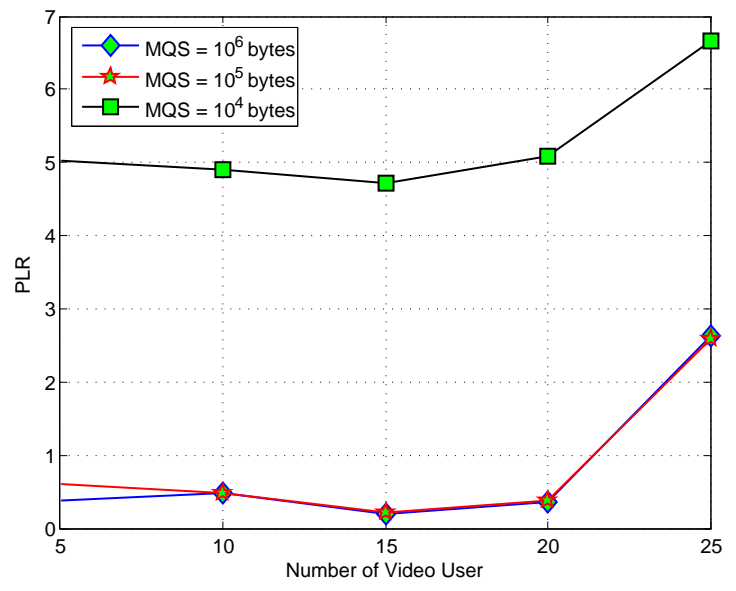

Fig. 15. The effect of the MQS on the PLR of Video flow

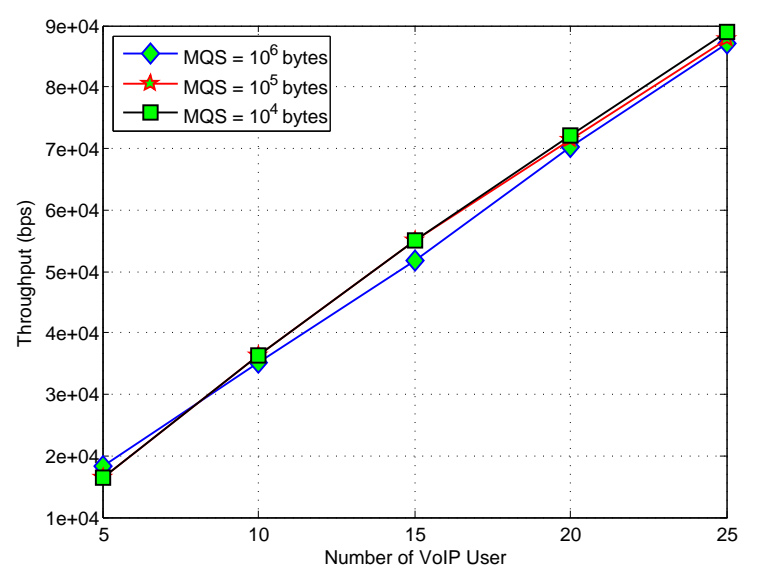

Fig. 16. The effect of the MQS on the cell throughput of VoIP flow

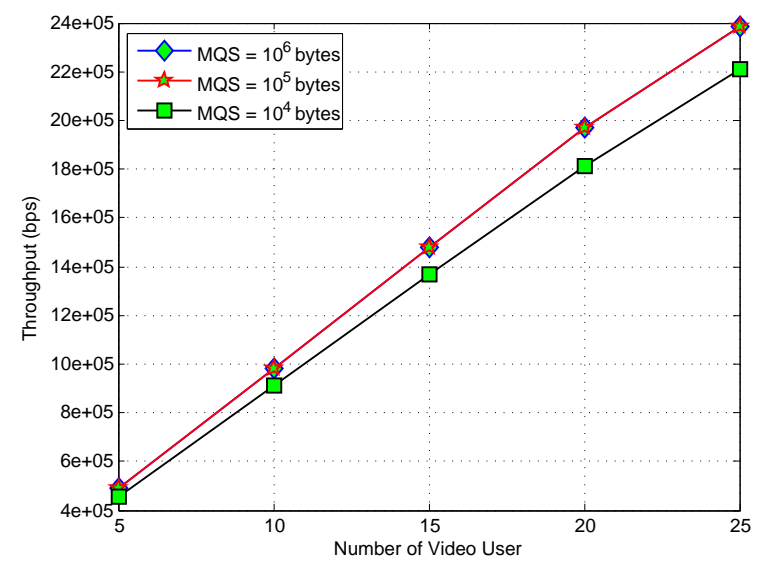

Fig. 17. The effect of the MQS on the cell throughput of Video flow

\section{CONCLUSION}

In this paper, we propose a new Channel- and QoS-Aware scheduling scheme for the downlink direction in LTE network. The main idea in the scheduler is the consideration of the MQS factor into the priority metric The metric is the combination of the MQS factor and the metrics of the M-LWDF, VT-MLWDF and Queue-HOL-MLWDF schedulers with essential modifications. The simulation results show that the proposed scheduler not only meets QoS requirements for real-time services but also outperforms the M-LWDF, VT-MLWDF and Queue-HOLMLWDF schedulers in terms of PLR, cell throughput, FI and SE, especially for Video flow. The proposed scheduler also improves significantly the delay in comparison with the VT-MLWDF and Queue-HOL-MLWDF schedulers ones for both VoIP and Video flows. It can be said that when considering the MQS as a factor for the metric in the proposed scheduler, the system performance is improved significantly, specially for Video flow. Therefore, it can be concluded that the proposed scheduler is very suitable for real-time services such as VoIP, Video, etc. for the downlink direction in LTE system.

In this study, we have not yet considered the presence of besteffort flow. They might bring new directions to evaluate the proposed scheduler in heterogeneous traffic in LTE network.

\section{REFERENCES}

[1] 3GPP. http://www.3gpp.org

[2] 3GPP. Requirements for Evolved UTRA (E-UTRA) and Evolved UTRAN (E-UTRAN). TR 25.913, 3rd Generation Partnership Project (3GPP), 122009.

[3] 3GPP. Evolved Universal Terrestrial Radio Access (EUTRA); Physical channels and modulation. TS 36.211, 3rd Generation Partnership Project (3GPP), 032010.

[4] Mehdi Alasti, Behnam Neekzad, Jie Hui, and Rath Vannithamby. Quality of service in wimax and lte networks [topics in wireless communications]. Communications Magazine, IEEE, 48(5):104-111, 2010.

[5] Salman Ali and Muhammad Zeeshan. A utility based resource allocation scheme with delay scheduler for lte service-class support. In Wireless Communications and Networking Conference (WCNC), 2012 IEEE, pages 14501455. IEEE, 2012.

[6] Pablo Ameigeiras, Jeroen Wigard, and Preben Mogensen. Performance of the m-lwdf scheduling algorithm for streaming services in hsdpa. In Vehicular technology conference, 2004. VTC2004-Fall. 2004 IEEE 60th, volume 2, pages 999-1003. IEEE, 2004.

[7] Francesco Capozzi, Giuseppe Piro, Luigi Alfredo Grieco, Gennaro Boggia, and Pietro Camarda. Downlink packet scheduling in lte cellular networks: Key design issues and a survey. Communications Surveys \& Tutorials, IEEE, 15(2):678-700, 2013.

[8] Dakuri Chiranjeevi, V Mahender, B Kiran, and B Raja Kumar. Channel aware scheduling algorithms for 3gpp lte downlink.

[9] Chen-Nee Chuah and Randy H Katz. Characterizing packet audio streams from internet multimedia applications. In Communications, 2002. ICC 2002. IEEE International Conference on, volume 2, pages 1199-1203. IEEE, 2002.

[10] Mauricio Iturralde, Tara Ali Yahiya, Anne Wei, and AndreLuc Beylot. Performance study of multimedia services using virtual token mechanism for resource allocation in lte networks. In Vehicular Technology Conference (VTC Fall), 2011 IEEE, pages 1-5. IEEE, 2011.

[11] Richard Musabe and Hadi Larijani. Cross-layer scheduling and resource allocation for heterogeneous traffic in $3 \mathrm{~g}$ 
lte. Journal of Computer Networks and Communications, 2014, 2014.

[12] Moustafa M Nasralla and Maria G Martini. A downlink scheduling approach for balancing qos in lte wireless networks. In Personal Indoor and Mobile Radio Communications (PIMRC), 2013 IEEE 24th International Symposium on, pages 1571-1575. IEEE, 2013.

[13] Giuseppe Piro, Luigi Alfredo Grieco, Gennaro Boggia, Francesco Capozzi, and Pietro Camarda. Simulating lte cellular systems: an open-source framework. Vehicular Technology, IEEE Transactions on, 60(2):498-513, 2011.

[14] Giuseppe Piro, Luigi Alfredo Grieco, Gennaro Boggia, Rossella Fortuna, and Pietro Camarda. Two-level downlink scheduling for real-time multimedia services in lte networks. Multimedia, IEEE Transactions on, 13(5):10521065, 2011.

[15] M. Reisslein, L. Karam, and P. Seeling. H. 264/AVC and SVC Video Trace Library: A Quick Reference Guide http://trace. eas. asu. edu. 2009
[16] Jong-Hun Rhee, Jack M Holtzman, and Dong-Ku Kim. Scheduling of real/non-real time services: adaptive exp/pf algorithm. In Vehicular Technology Conference, 2003. VTC 2003-Spring. The 57th IEEE Semiannual, volume 1, pages 462-466. IEEE, 2003.

[17] Bilal Sadiq, Ritesh Madan, and Ashwin Sampath. Downlink scheduling for multiclass traffic in lte. EURASIP Journal on Wireless Communications and Networking, 2009:14, 2009.

[18] Dardouri Samia and Bouallegue Ridha. A new scheduling algorithm for real-time communication in lte networks. In Advanced Information Networking and Applications Workshops (WAINA), 2015 IEEE 29th International Conference on, pages 267-271. IEEE, 2015.

[19] Freescale Semiconductor. Long term evolution protocol overview. White Paper, Document No. LTEPTCLOVWWP, Rev 0 Oct, 2008. 